\title{
Robust Bio-Inspired Routing Protocol in MANETs using Ant Approach
}

\author{
Arush Sharma and Dongsoo S. Kim \\ Department of Electrical and Computer Engineering \\ Indiana University Purdue University, Indianapolis IN 46202, USA \\ \{sharmaas, dskim\} @iupui.edu
}

\begin{abstract}
This paper discusses about developing a routing protocol for mobile ad-hoc networks in a bio-inspired manner. Algorithms inspired by collective behavior of social insect colonies, bird flocking, honey bee dancing, etc., promise to be capable of catering to the challenges faced by tiny wireless sensor networks. We propose an energy efficient multi-path routing algorithm based on foraging nature of ant colonies. This paper considers many meta-heuristic factors to provide good robust paths from source node to destination node in a hope to overcome the challenges posed by resource-constrained sensors. The performance of proposed ant colony routing algorithm is compared against well-known AODV routing protocol.
\end{abstract}

Keywords: Ant Colony Optimization, Swarm Intelligence, Wireless Sensor Networks, Mobile Ad-Hoc Networks.

\section{$1 \quad$ Introduction}

Wireless Sensor Networks (WSN) consist of a large number of nodes equipped with sensing capabilities; communication interfaces which has limited memory and energy resources. WSN nodes are statically deployed over large areas. However, they can also be mobile interacting with the environment. WSNs have wide spectrum of applications which includes environmental sensing, health care, traffic control, tracking of wild life animals, etc. Usually individual sensor nodes send their data towards base station node (commonly known as sink node). Intermediate nodes perform relaying of sensed data towards the destination.

\subsection{Design Challenges}

Following are the design challenges faced by WSNs Routing Protocols.

1. Low Computational and memory requirements.

Sensor nodes are equipped with a low end CPU and have limited memory. Therefore, it is mandatory that the routing algorithm has minimum overhead to make its execution feasible and effective.

This is the author's manuscript of the article published in final edited form as:

Sharma A., Kim D.S. (2019) Robust Bio-Inspired Routing Protocol in MANETs Using Ant Approach. In: Lee S., Ismail R., Choo H. (eds) Proceedings of the 13th International Conference on Ubiquitous Information Management and Communication (IMCOM) 2019. IMCOM 2019. Advances in Intelligent Systems and Computing, vol 935. Springer, Cham https://doi.org/10.1007/978-3-030-19063-7_9 


\section{Self-organization}

Wireless Sensor Network is expected to remain active for considerable period of time. Within that time period, few new nodes might be added to the network, while other nodes might die due to energy depletion or may become operational. A routing protocol therefore should be robust to such dynamic and unpredictable events. It should be empowered with self-organizing properties to let the network function as an autonomous system.

\section{Energy Efficiency}

Nodes are equipped with small non-rechargeable batteries. Therefore, the efficient battery usage of a sensor node is very important aspect to support the extended operational lifetime of network. The routing protocol is expected to forward the data packets across multiple paths, so that all nodes can deplete their battery source at a comparable rate. This results in load balance of network and increases the network lifetime.

\section{Scalability}

In WSN applications, thousands of nodes are generally deployed that have short communication ranges and high failure rates. Hence a routing protocol should be able to cope with the above challenges.

It is imperative to design such routing protocols which can cater to the aforementioned challenges and are robust and adaptive. Nature provides us examples of mobile, independently working agents which seamlessly work together to perform tasks efficiently, for example, flight of migratory birds, ant colony optimization, etc. Nature inspired algorithms also known as Swarm Intelligence (SI) are based on collective behavior of social insect colonies and other animal societies for solving different types of communication problems.

The remainder of paper is given as follows. At first general introduction and challenges are given which gives us the motivating factors to do research. Section 2 discusses about the related work done in the field of ant routing algorithms. Section 3 talks about the novel ideas implemented in ant routing algorithm. Section 4 does a comparison study against AODV routing protocol. Finally we conclude by giving a short summary and an outlook on future work.

\subsection{Background}

Ant algorithms are special class of SI algorithms, which consist of population of simple agents (ants) which interact locally and with their environment. The foraging behavior of ant colony inspires Ant Colony algorithm. Initially, ants randomly wander around the nest searching for food. When the food is found, they take it back to the colony and leave a trail of pheromones on the way. Other ants can sense the phero- 
mone concentration and prefer to follow directions with higher pheromone density. Since shorter paths can be traversed faster, they will eventually outweigh the less optimal routes in terms of pheromone concentration. Additionally, pheromones evaporate over time, so ants are less likely to follow an older path which makes them search for newer paths simultaneously. In a case where an obstacle gets in their way, ants again initiates the route discovery process by randomly selecting the next hop until the ants converge on the paths with relatively higher concentration of pheromone.

\section{$2 \quad$ Related Work}

AntNet proposed by Di Caro and Dorigo [1] is a routing technique which is applied for best-effort IP networks. Optimizing the performance of entire network is its main aim according to the principles of Ant Colony Optimization, AntNet is based on a greedy stochastic policy, where each node maintains a routing table and an additional table containing statistics about the traffic distribution over the network. The routing table maintains for each destination and for each next hop a measure of the goodness of using the next hop to forward data packets to destination. These goodness measures, called pheromone variables, are normalized on the stochastic policy. This algorithm uses forward ants and backward ants to update the routing table. The forward ants use heuristic based on the routing table to move between a pair of given nodes and are used to collect information about the traffic distribution over the network. The backward ant stochastically follows the path of forward ants in reverse direction. At each node, the backward ant updates the routing table and the additional table which contains traffic statistics of the network.

The energy-efficient ant-based routing algorithm (EEABR) is a routing protocol for WSNs and extends AntNet proposed by Tiago Camilo et. al., [2]. EEABR tries to minimize memory requirements as well as the overall energy consumption of the original AntNet algorithm. The ants retain information of only last two visited nodes because it takes into account the size of ant packet to update pheromone trail. In the typical ant-based algorithm, each ant carries the information of all the visited nodes. Then, in a network consisting of very large number of sensor nodes, the size of information would cause considerable energy to send ants through the network. Each node keeps the information of the received and sent ants in its memory. Each memory record contains the previous node, the forward node, the ant identification, and a timeout value. The transmission probability considers the artificial pheromone value and the remaining energy of the possible next hop.

Ladder Diffusion Algorithm proposed by Ho et al. [3] addresses the energy consumption and routing problem in WSNs. The algorithm tries to reduce the energy consumption and processing time to build the routing table and avoid the route loop. In this algorithm, the sink node broadcasts the ladder creating packet with the grade value of one. The grade value of one means that the sensor node receiving this ladder 
creating packet transmits data to the sink node requires only one hop. Then sensor nodes increments the grade value of ladder creating packet and broadcast the modified ladder-creating packet. A grade value of two means that the sensor node receiving this ladder-creating packet sends data to the sink node requires two hop counts. And this step repeats until all the sensor nodes get the ladder-creating packet. The ladder diffusion algorithm assures that the direction of data transfer always occurs from a high grade value to a low grade value, which means each relay is forwarded to the sink node since each sensor node records the grade value of relay nodes in the ladder table. The path decision is based on the estimated energy consumption of path and the pheromone.

Energy-Aware Ant Routing in Wireless Multi-Hop Networks proposed by Michael Frey et. al., [4] provides new mechanisms for estimating the fitness of a path and energy information dissemination thus enabling to prolong the network lifetime. The network lifetime is the time span a network can fulfill its service. Traditional Ant Routing Algorithm considers the pheromone value in its probabilistic routing decision process. This approach favours shortest paths over non-shortest paths which is not suitable for energy constrained networks. EARA extends the ant routing algorithm with an energy heuristic for determining the nodes residual energy and scheme for estimating a path's energy. EARA algorithm considers the residual energy of a node as an additional heuristic. Since the residual energy of a node changes over time, periodic Energy Ants are released for updating the energy values in the node's routing table. Periodic Energy Ants are sent occasionally as it can be a costly operation in terms of consumed energy.

Ant Colony and Load Balancing Optimizations for AODV Routing Protocol proposed by Ahmed M. Abd Elmoniem et al., [5] discusses about improving the AODV routing protocol by taking the Ant Colony Optimization approach. Forward ant agents are sent as a part of route establishment request to find the route to destination. This route establishment phase is very much similar to Route Request (RREQ) phase of AODV routing protocol except for the fact that if the route to destination doesn't exist and there exist no neighbour, then the ant is broadcasted. Otherwise, if the active neighbour exists with highest pheromone, the forward ant is sent to that neighbour. In case of destination node receiving forward ant, backward ant is sent to the source node with a route to destination which comes under the part of Route establishment reply phase. The pheromone update policy is applied on the nodes receiving backward ants. Also it is applied differently depending upon whether the node is an source node or intermediate node or is destination node. Once the source node receives backward ant, the Data transmission phase begins. Each node receiving data packets forwards it to neighbor according to the pheromone values. Neighbor node having greater pheromone receives more data than those having less pheromone which leads to load balancing. If the route doesn't exist at all, a Route Error (RERR) packet is sent to the source node. If the routing table entry of the destination doesn't exist in source node, it deletes the route and again initiates the route discovery process. 
Ant Colony Optimization for Routing and Load-Balancing: Survey and New Directions presented by Kwang Mong Sim et al., [6] provides comparison of the approaches for solving the convergence problem in ACO algorithms. When the network reaches its equilibrium state, the already discovered optimal path is given more preference over other paths by the ants which leads to many problems such as congestion, reduction of probability for selecting other paths, network failure, etc. In order to mitigate this problem, some of the approaches include evaporation, aging, pheromone smoothing and limiting, privileged pheromone laying etc. Evaporation of pheromone is a technique to prevent the ants of favoring the older or stale paths which makes an ant to concurrently search for fresh paths. Aging refers to quantity of pheromone deposited by the ant. Older ant will deposit less pheromone compared to its young contemporary since they take more time in reaching destination. Limiting and Smoothing Pheromone refers to limiting the pheromone deposit by placing an upper bound which reduces preference of optimal paths over non-optimal paths In privileged pheromone laying, only certain ants are permitted to deposit extra pheromone. This makes the ant to converge to a solution by taking less time.

Ant-routing-algorithm (ARA) for mobile multi-hop ad-hoc networks- new features and results explored by Mesut Gunes et al., [7] is based on ant algorithms which makes it highly adaptive and efficient. The routing algorithm consists of three phases. Route Discovery Phase requires use of forward ant (FANT) and backward ant (BANT) control agents. FANT establishes the pheromone trail back to the source node. Similarly BANT establishes pheromone track back to the destination node. Node receiving FANT for the first time creates an entry in its routing table consisting of destination address which is the origin of FANT, next hop which is address of the previous node from which it received FANT and pheromone value which is computed based on the number of hops the FANT took to reach the node. The node forwards the FANT to its neighbors. Once the destination node receives FANT, it sends BANT back to the source node. Once the source nodes receives BANT from the destination node, the path is established and data packets can then be sent which comes under the Route Maintenance Phase. When data packets are relayed to destination by a node, it increases the pheromone value of the routing table entry. The last phase of ARA handles the routing failure caused by the mobility of node which are very common in MANETs. ARA assumes IEEE 802.11 on the MAC layer which enables routing algorithm to recognize the failure of route through a missing acknowledgement on the MAC layer. Node deactivates the link by setting the pheromone value to 0 . The node then searches for an alternative link in its routing table. If there exist a route to destination in its routing table, it sends the packet via this path. If there exist multiple entries in the routing table, the node will not send any data packets. Instead it informs the source node which has to initiate the route discovery process again.

\subsection{Working of Traditional Ant Colony Based Routing Algorithm}

The basic ACO takes a reactive probabilistic approach of finding good robust paths between source and destination. The algorithm follows: At regular intervals, a forag- 
ing ant is launched with a mission to find a path to destination. This establishes backward pheromone trail from destination to source. When a node receives a foraging ant for the first time, it creates an entry in its routing table. The entry consists of destination address which is the source address of the foraging ant, next hop which implies the node from which it received the packet, and pheromone value. The foraging ant probabilistically selects the next hop to reach destination. Duplicate foraging ants are then removed by identifying their unique sequence ID. Once a forward ant reaches its destination, it triggers the flooding of backward ants which the destination will send to the source node. The backward ant establishes the pheromone trail from source to destination. After calculation of the selection probabilities, the node will forward the data packets to that neighbor node which has relatively higher selection probability among its neighbor set. The data packet is sent to the selected relayed node and is further relayed towards the destination node. The selected relay nodes increments their pheromone value by a specific amount. Like their natural counterpart, artificial pheromones decay over time. The evaporation process provides a negative feedback in the system which helps ant avoid the stale paths in the network. The evaporation of pheromones takes place constantly by decreasing the pheromone values. The procedure finishes once the data packet reaches the destination node. Nodes maintain the neighbor entity in its neighbor table by sending Hello Packet periodically to each other. Hello packet sending interval can be different according to different mobility scenarios. If a node doesn't receive the neighbor's Hello packet after certain period of time, it then deletes neighbor information from its neighbor table.

\section{Proposed Idea}

This section discusses about various heuristic factors which are considered in the proposed routing algorithm which makes it novel compared to already existing routing algorithms for MANETs.

Pheromone and Repellent Pheromone: Ants in nature while travelling from their nest to food source make the routing decision when they reach intersection, i.e., when more than one path is available for their next hop. In such scenario, the probability to choose that path is more which has relatively higher concentration of pheromone compared to other available paths. After the robust path is established, the ants continue to use that path until they encounter some obstacle, for example, placing a stone or water coming from some source accidentally flowing through the path recently formed. In such case, ants no longer use that path and instead begin exploring new paths. Pheromones with repellent property are deposited by ants so that their contemporaries no longer use the earlier efficient path. This property if incorporated in algorithm would make delay less in the network as the nodes would be aware of failed paths in the network.

RSSI: In WSN, sensor nodes are aware of the proximity of their neighbors through RSSI. If the scenario is considered where the source node and destination node are 
placed very far apart such that the destination node barely comes under the transmission range of source node, both the nodes will receive packets with very low RSSI as power of received signal decreases with increase in distance. As a result the chances of packet drops are very high. But due to less number of hops between source and destination node, the destination node will experience less delay. Whereas if source node and destination node are connected such that there exist neighboring nodes through which the packets can be transferred in a multi hop fashion, then the nodes will receive packets with very high value of RSSI due to close vicinity with each other. Nevertheless, the delay experienced by the packet will be more as the packet would have travelled with more number of hops from source to destination.

Therefore, it is understood that extreme values of RSSI is not good for our proposed system. With the logic of RSSI explained above, it is vital that goodness of RSSI closely follows the Gaussian distribution [9] as shown in the figure below.

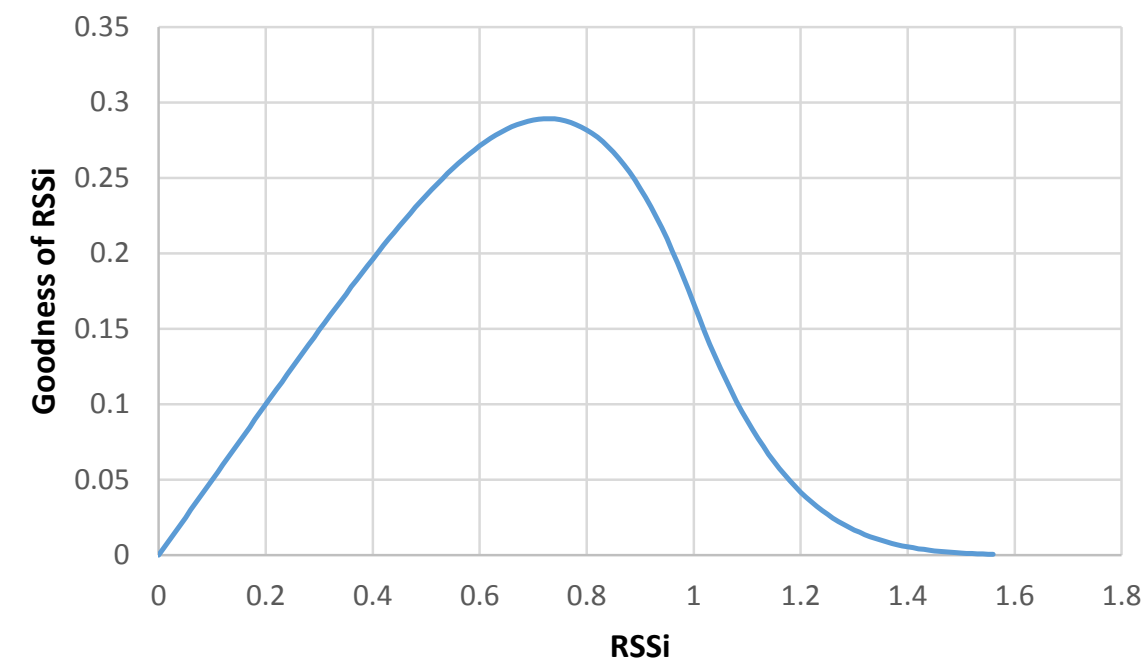

Fig. 1. Goodness of RSSI against varying range of RSSI

With reference to [12], if two nodes (source and destination) are placed such that the distance between them is less than the transmission radius $\mathrm{R}$, the total expected hop count from source node to destination node is given by

$$
\frac{d}{x}\left[\frac{1}{\left[p(x)\left(1-(1-p(x))^{u}\right)\right]}+\frac{u}{\left[\left(1-(1-p(x))^{u}\right)\right]}\right]
$$

where $\mathrm{x}$ is the distance between two consecutive nodes. $p(x)$ is the probability of receiving packet and $\mathrm{u}$ is a constant and is selected as 1 . 
$(x)$ is dependent on several measurements such as signal strengths, delay, etc. It is approximated by following equation:

$$
P(x)=\left\{\begin{aligned}
1-\left(\left(\frac{x}{R}\right)^{2 \beta} / 2\right), & x<R \\
((2 R-x) / R)^{2 \beta} / 2, & x \geq R
\end{aligned}\right.
$$

Where $\beta$ is constant and is selected as 2 .

In the experiment, $\mathrm{R}$ is selected such that the packet delivery ratio at destination node is $80 \%$.

In order to make the equation 1 independent of particular distance $d$, it is optimized which is given as follows:

$$
h(x, u, \beta, R)=\frac{R}{x}\left[\frac{1}{\left[p(x)\left(1-(1-p(x))^{u}\right)\right]}+\frac{u}{\left[\left(1-(1-p(x))^{u}\right)\right]}\right]
$$

By plugging in the value of $u$ in equation 3 , it is simplified by following equation

$$
h(x)=\frac{R(1+p(x))}{x p^{2}(x)}
$$

Taking the inverse of equation (4), Goodness of RSSI is achieved which follows the graph in figure 1 . Hence

$$
f(S)=\frac{1}{h(x)}=\frac{R(1+p(x))}{x p^{2}(x)}
$$

Where $\mathrm{f}(\mathrm{S})$ is known as Goodness RSSI

After the goodness of RSSI is calculated, the probabilistic formulae and goodness RSSI follows linear relationship.

In proposed routing algorithm, signal to noise ratio is computed by default at physical layer (with reference to OSI model). As the layers in OSI model are independent of each other, the Network layer (Layer 3) will not be aware of this parameter. Hence, it is imperative to add a packet tag at physical layer. The packet tag consists of signal to noise ratio information in $\mathrm{dBm}$ units. With respect to Network layer mechanism, when a node receives a hello packet from its neighbor, it scans for the packet tag so that it is aware of its neighbor's received strength. Each node then maintains a neighbor table where RSSI records are maintained against every neighbor. Also it is widely known that RSSI fluctuates too often even when nodes are static [10], the exponential moving weighted average approach chosen by us helps in smoothening the RSSI value. 
Residual Energy: If the nodes among the discovered robust path are going to be used extensively for data packet transmission, their battery will deplete faster compared to nodes on non-efficient paths. This will result in creation of void nodes in the network which may lead to network partition. Inclusion of residual energy of node [11] in the routing decision will help in exploring paths other than already discovered robust paths. This technique will improve the lifetime of the network.

Proposed routing algorithm is an extended version of traditional ant colony based routing algorithm in which the main objective is to maximize the network lifetime. Traditional ant colony routing algorithm considers the pheromone value in its probabilistic routing decision process. This case is however not favorable for energy constrained networks. With additional heuristics discussed above, the extended probabilistic formula shall look like:

$$
P_{n, d}=\frac{\left(\tau_{n, d}^{i}\right)^{\alpha}\left(h_{n, d}^{i}\right)^{-\beta}\left(E_{n}^{i}\right)^{\delta}\left(f(S)_{n}^{i}\right)^{\gamma}}{\sum_{j \in N_{i}}\left(\tau_{j, d}^{i}\right)^{\alpha}\left(h_{j, d}^{i}\right)^{-\beta}\left(E_{j, d}^{i}\right)^{\delta}\left(f(S)_{j, d}^{i}\right)^{\gamma}}(6)
$$

where $\mathrm{n}$ is the next hop selected by an ant to reach destination $\mathrm{d}$ from node $\mathrm{i}, \tau_{\mathrm{n}, \mathrm{d}}^{\mathrm{i}}$ is a pheromone value from neighbor $\mathrm{n}$ of node $\mathrm{i}$ to destination. $\mathrm{h}$ is the number of hops taken by an ant to reach destination from node i. $N^{i}$ is the set of neighbors of node i. E is the remaining energy in the node, $\mathrm{f}(\mathrm{S})$ is the goodness RSSI which follows Gaussian distribution as shown in figure $1 . \alpha, \beta, \delta, \gamma$ are the factors to adjust the relative importance of pheromone concentration, hops, residual energy, and goodness RSSI respectively.

As discussed in section 2.1, nodes receiving the ants update the pheromone value in their routing table by depositing a constant value of pheromone in their routing table which acts as a positive feedback. As a result, an impulsive response is observed with regards to pheromone whenever a node receives an ant. Similar to the biological ants, the pheromone value is a function of time which means pheromone value decreases exponentially as the time progresses which makes it volatile.

\section{$4 \quad$ Results}

In this section, the experimental setup is discussed, followed by performance metrics and also simulation results.

\subsection{Experimental Setup}

The simulations are carried out using well known discrete event network simulator known as Network Simulator 3 (NS-3). In the simulation, the static nodes are deployed in a 10X10 grid with a distance of 50 meters apart. As shown in Figure 2, source and destination pair is indicated by blue color. Each node is configured with Friis Propagation Loss Model with respect to physical layer in which the transmission range of node can be varied by adjusting the receiver's sensitivity. Each node is in- 
stalled with a battery of capacity 800 Joules. Approximately 4900 data packets are generated by the source node and are sent to the destination node. The Simulation runs for 500 seconds. With this experiment setup, the performance of Ant Colony Based Routing is compared against AODV Routing Protocol.

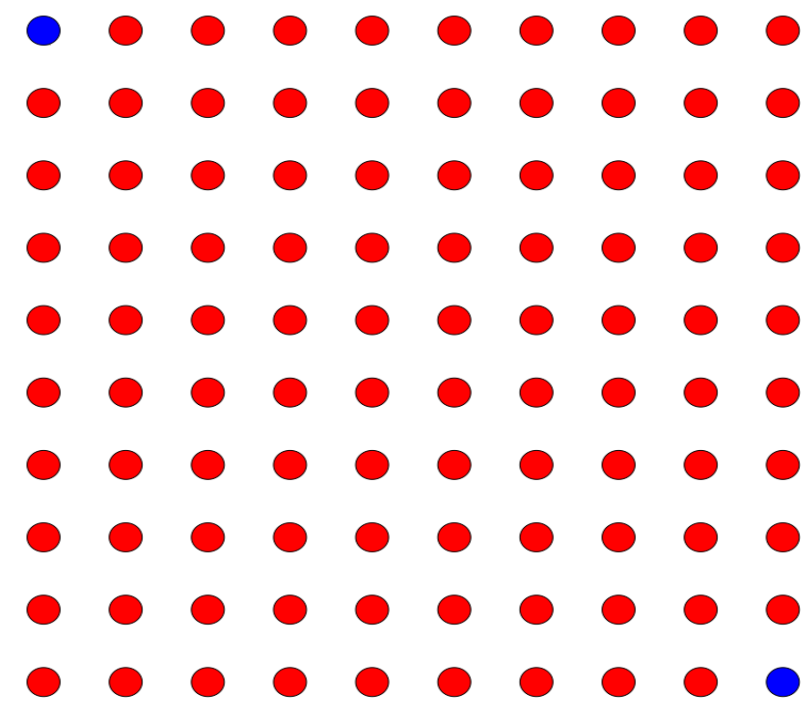

Fig. 2. Topology of Static Nodes in Network

\subsection{Performance Metrics}

The following performance metrics are considered for the evaluation of Ant Colony Based Routing Algorithm and AODV Routing Protocol:

- Throughput

The rate at which the data packets are delivered successfully by the network from a source to destination is known as throughput. It is also known as goodput. It is represented by bits or bytes per second. The throughput is affected by various factors such as limitation of physical medium, processing power, end user behavior etc. A higher throughput is what is desired in any communication based network.

- Mean Delay

It is the time consumed by the data packet to reach the destination. The mean delay was calculated [8] by computing the ratio of sum of all end-to-end delays for all received packets of the flow to the total number of received packets.

- Network Lifetime 
It is defined as the time span a network can fulfill its service whereby source node and destination node communicate with each other by exchanging the data packets and other control packets.

\subsection{Simulation Results and Analysis}

The performance of both the routing protocols is analyzed under these three scenarios:

a) Number of Hops

In this scenario multiple source and destination pairs are selected at random which gives us an idea of the protocol performance against change in number of hops between source and destination.

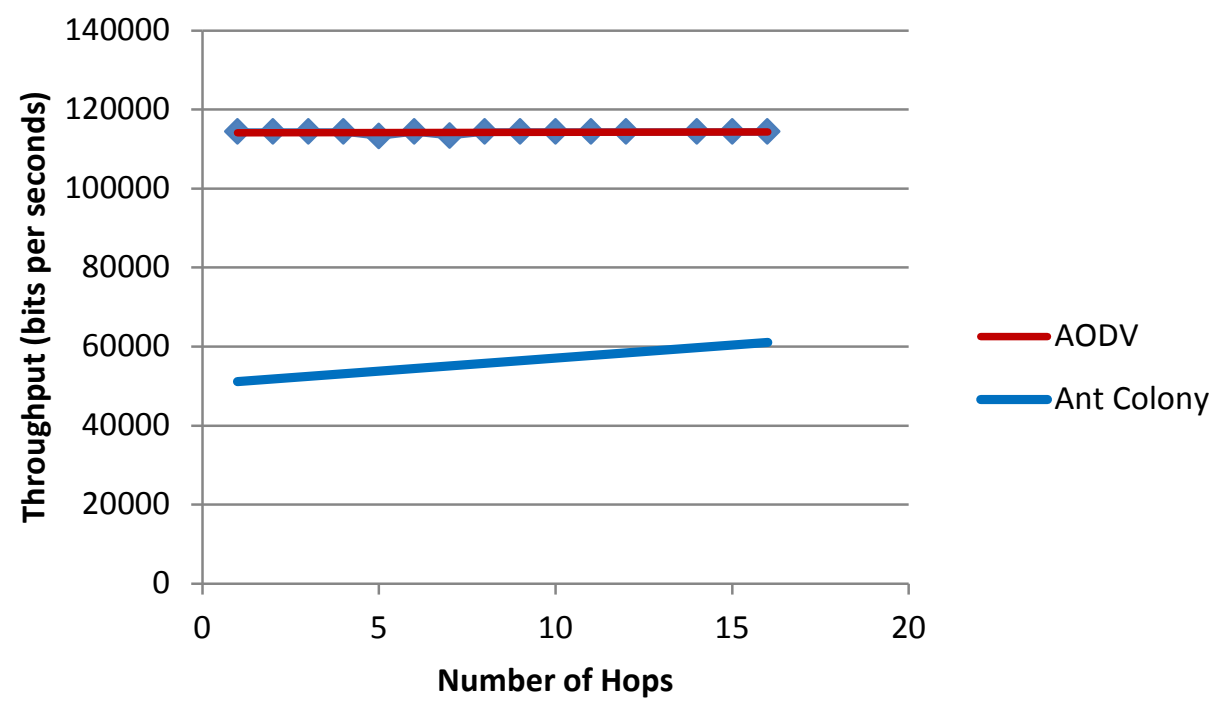

Fig. 3. Throughput versus Number of Hops

It is observed that AODV outperforms Ant Colony Based Routing Algorithm because Ant Colony Based Routing Algorithm has randomness property in terms of selecting the neighboring nodes. As a result, not all decisions would be favorable. Whereas in AODV, there are no multiple paths between source and destination which makes it more optimal in terms of hop counts. 


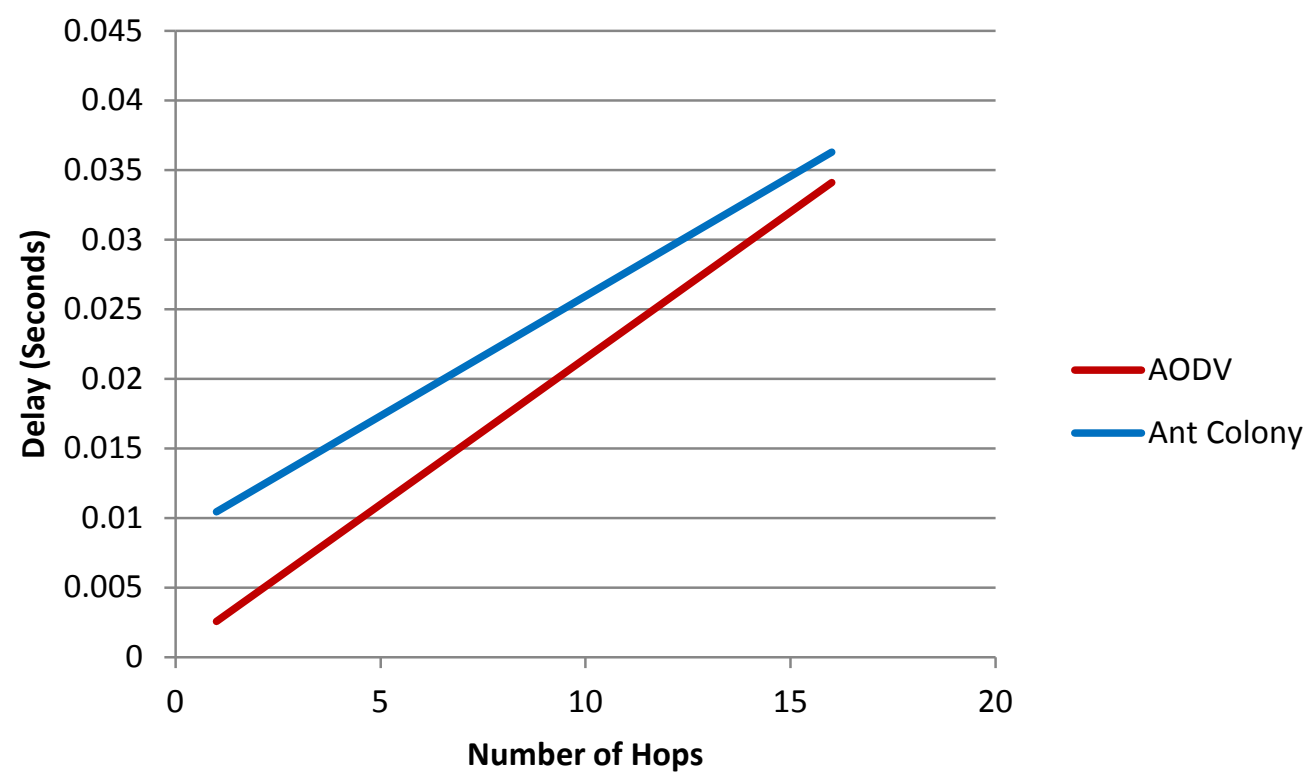

Fig. 4. Delay vs Number of Hops

As expected the increase in delay is caused by increase in number of hops between source and destination. In Ant Colony Algorithm, due to the property of randomness in path selection, its performance lacks in comparison with AODV.

\section{b) Background Traffic}

Several source and destination node pairs are selected at random in the simulation which generates UDP traffic. The logic of using Background Traffic is to know how the algorithm performs in a real-life network environment where there is a disturbance along with regular traffic flow. 


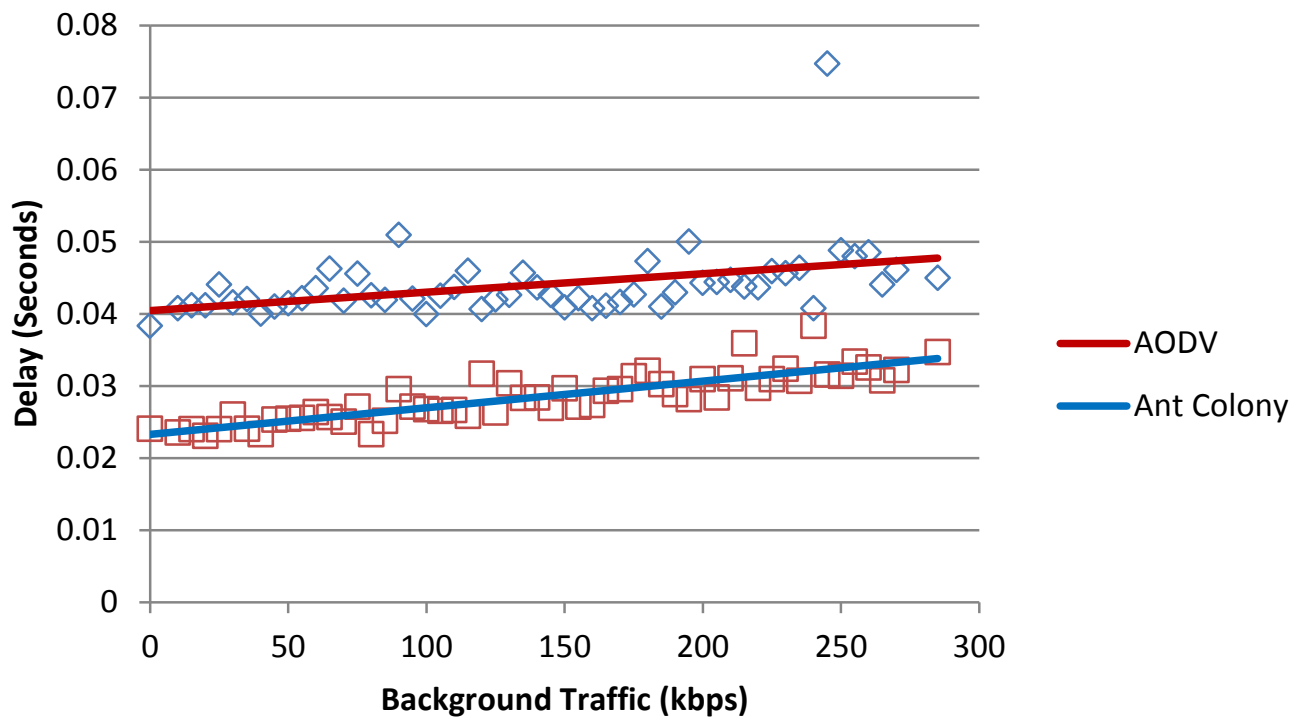

Fig. 5. Delay against increase in Background Traffic

Ant Colony Routing experiences less delay against AODV. This is due to the fact that whenever node faces congestion due to increase in background traffic, due to randomness property, the packet is received by node which has relatively less delay and is relayed to the destination node. Whereas in AODV, since there is no multipath routing, the delay will be more. 


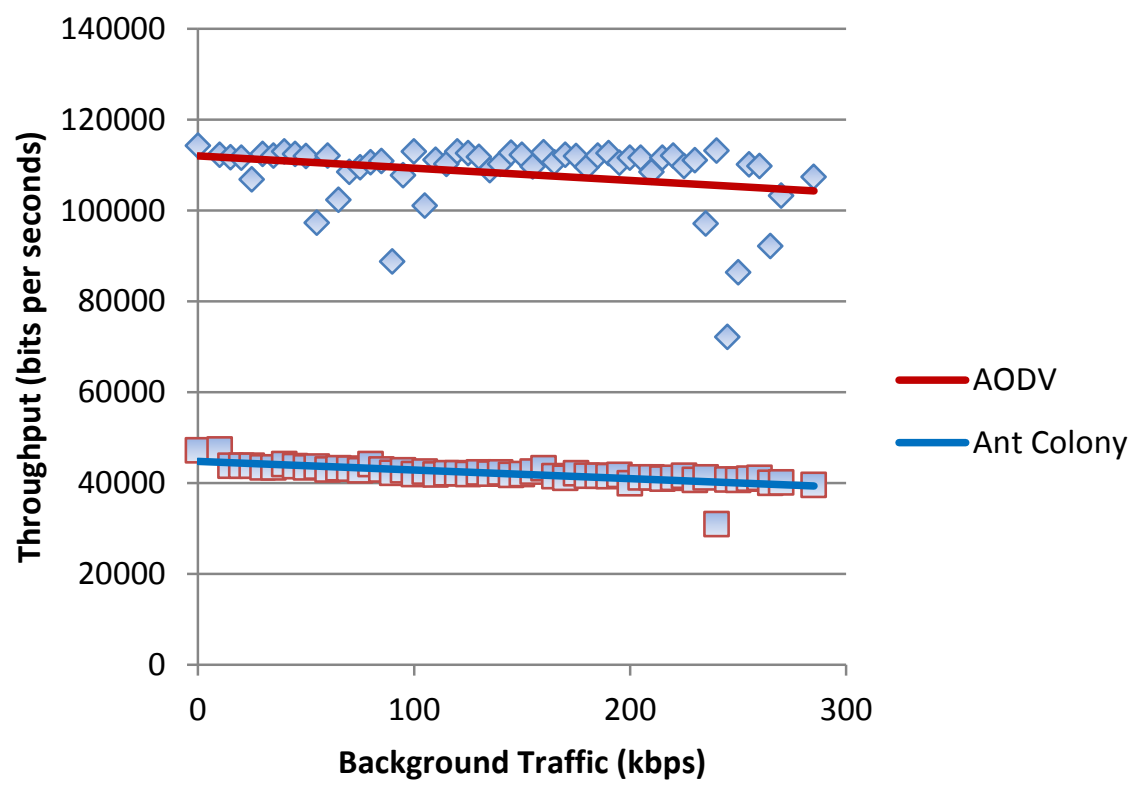

Fig. 6. Throughput against increase in Background Traffic

In both the cases, due to increase in background traffic, decrease in throughput is expected. However Ant Colony Routing Algorithm experiences more packet drop ratio in comparison to AODV Routing Protocol which makes its throughput performance lesser than AODV.

c) Network Lifetime 


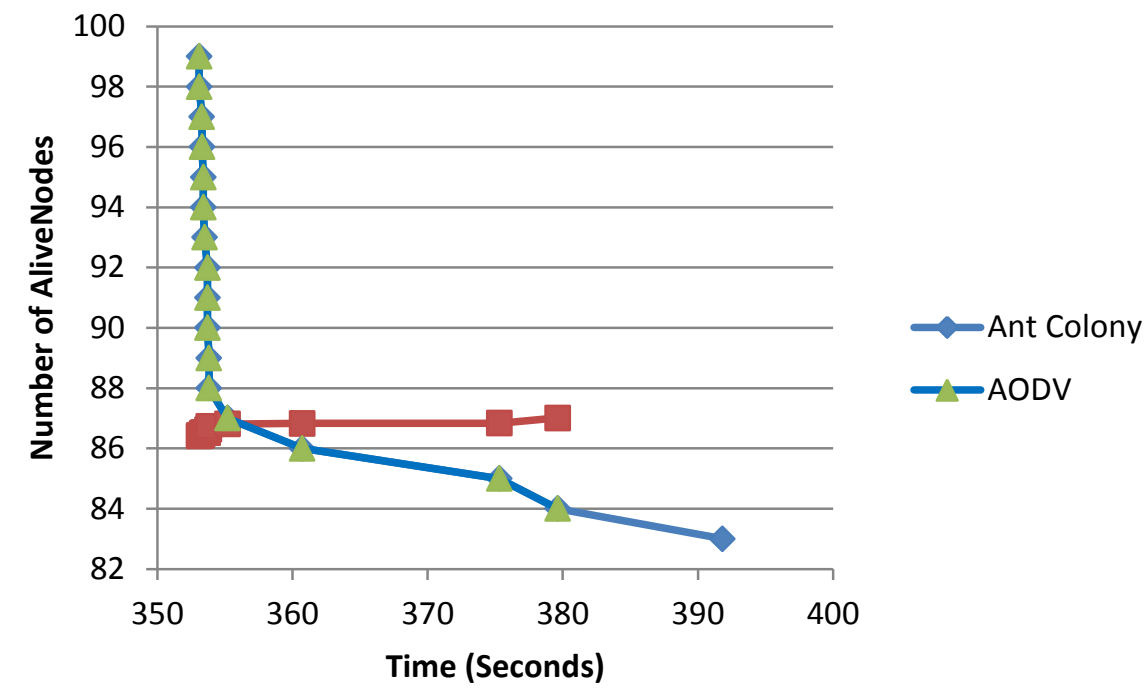

Fig. 7. Lifetime of Network

Ant Colony Routing outperforms AODV in terms of Network Lifetime due to the fact that energy based robust routing is implemented in layer 3. As a result, its performance is better than AODV since AODV is not energy aware routing protocol.

\section{Conclusion and Future Work}

This work sought to develop a routing protocol for wireless sensor networks/MANETs in NS-3. We analyzed the performance of network by considering the basic performance metrics such as throughput, delay, network lifetime and compared against AODV routing protcol. In future we would include the repellent pheromone phase in our routing protocol so that the nodes could detect the failure of its neighbor on the IEEE 802.11 MAC layer. We will include various test scenarios in terms of mobility of the network to test its robustness.

\section{References}

1. Gianni Di Caro and Marco Dorigo. Antnet: Distributed stigmergetic control for communication networks. Journal of Artificial Intelligence Research, 1998.

2. T. Camilo, C. Carreto, J. Silva, and F. Boavida. An energy-efficient ant-based routing algorithm for wireless sensor networks. Ant Colony Optimization and Swarm Intelligence, 
pages 49-59, 2006.Author, F., Author, S., Author, T.: Book title. 2nd edn. Publisher, Location (1999).

3. Jiun-Huei Ho, Hong-Chi Shih, Bin-Yih Liao, and Shu-Chuan Chu. A ladder diffusion algorithm using ant colony optimization for wireless sensor networks, 2012.

4. Michael Frey, Friedrich Große and Mesut Gunes. Energy-Aware Ant Routing in Wireless Multi-Hop Networks, 2014.

5. Ahmed M. Abd Elmoniem, Hosny M. Ibrahim, Marghny H. Mohamed and Abel-Rahman Hedar. Ant Colony and Load Balancing Optimizations for AODV Routing Protocol. Sensor Networks and Mobile Computing, 2011.

6. Kwang Mong Sim and Weng Hong Sun. Ant Colony Optimization for Routing and LoadBalancing: Survey and New Directions. IEEE Transactions on Systems, Man, and Cybernetics-Part A: Systems and Humans, Vol. 33, No. 5, September 2003.

7. Mesut Gunes, Martin Kahmer, Imed Bouazizi. Ant-Routing-Algorithm (ARA) for Mobile Multi-Hop Ad-Hoc Networks- New Features and Results. Proceedings of the Med-Hoc Net 2003 Workshop Mahdia, Tunisia 25-27 June, 2003.

8. Gustavo Carneiro, Pedro Fortuna, Manuel Ricardo. FlowMonitor- a network monitoring framework for the Network Simulator 3 (NS-3), 2009.

9. Zinon Zinonos, Vasos Vassiliou, Tasos Christofides. Radio Propagation in Industrial Wireless Sensor Network Envrionments: From Testbed to Simulation Evaluation, 2012.

10. Yogita Chapre, Prasant Mohapatra, Sanjay Jha, Aruna Seneviratne. Received Signal Strength Indicator and its Analysis in a Typical WLAN System. 38th Annual IEEE Conference on Local Computer Networks, 2013.

11. Soon-gyo Jung, Byungseok Kang, Sanggil Yeoum, and Hyunseung Choo. Trail-Using Ant Behavior Based Energy-Efficient Routing Protocol in Wireless Sensor Networks. International Journal of Distributed Sensor Networks. Volume 2016, Article ID 7350427, 9 pages.

12. Johnson Kuruvila, Amiya Nayak, and Ivan Stojmenovic. Hop Count Optimal PositionBased Packet Routing Algorithms for Ad Hoc Wireless Networks With a Realistic Physical Layer. 